\title{
Note technique sur le dispositif de Johnson pour le maintien à une humidité constante d'insectes d'élevage. Application éventuelle à un élevage de glossines
}

\author{
par G. MAILLOT
}

Johnson (1940) (1) a construit un appareil relalivement simple pour l'élevage d'insectes à un taux d'humidité constante. Un compresseur d'air débite, à la cadence de 10 litres à l'heure, de l'air à travers un mélange aux proportions fixées de glycérine et d'eau. L'air se charge d'humidité ef pusse à travers le réservoir en verre où les insectes sont maintenus dans des tubes de verre clos à chaque extrémité par du tulle. Suivant les proportions du mélange eauglycérine*, on obtient à une même température divers taux d'humidité relative, supérieurs à 75 p. 100 et relativement constants. Le mélange, dont la composition peut être appréciée rapidement au moyen d'un densimètre, n'a besoin d'être rectifié, en général, qu'au bout de 4 jours. Le réservoir de verre où sont mainten is les insectes a une capacité d'environ 1 litre. L'air est donc renouvelé plusieurs fois par heure.

L'élevage des glossines sous les tropiques. plus particulièrement en saison sèche. ou sous des climats de savane se heurte à de grosses difficultés quand il s'agit de réaliser des concitions très proches de celles du microclimat de haute humidité propre et à la puspe (au début et à la fin de la nymphose) et à la mouche nouveaunće; ces conditions sont souvent très difficiles

* Le poids spécifique de ce mélange varie arec la température mais un mélange donné fournii des humidités relatives très voisines dans un large intervalle de températures. A la température de $25^{\circ}$ centigrades une solution de glycérine à 35 p. 100 donnc une humidité relative de 85,2 p. 100.

Rev. Elev. Med. vet. Pays trop. 1962, 15, no 3.

Reçu pour publication : septembre 1962. à réaliser d'une façon permanente ou même temporaire.

On peut en général obtenir sous les tropiques une salle d'élevage relativement abritée de la chaleur, la température de cette salle ne devant pas excéder $30^{\circ}$. Les mouches adultes peuvent, avec des humidités pas trop élevées pour beaucoup d'espèces, y être maintenues en survie assez longtemps. Une évaporation forte ne nuit pas, au contraire, à leur alimentation, mais si l'humidité est très basse, même pour des espèces apparemment très xérophiles comme $G$. morsitans, la reproduction est très diminuée (effet qui peut d'ailleurs ne se manifester qu'au bout d'une ou plusieurs générations (cf.' observations d'Evens à Léopoldville pour G. fuscipes quanzensis et élevage de G. morsilans en Arrique Orientale).

Dans quelle mesure le dispositif de Johnson pourrait-il être utilisé pour l'élevage des glossines?

L'appareil décrit par Johnson ne peut être utilisé tel quel pour les glossines étant donné sa faible capacité et l'emploi de tubes de verre où sont maintenus les insectes.

L'emploi de la cage Roubaud (garnie de tulle aix mailles assez larges pour pouvoir laisser pcsser la larve) est préférable. Une cage peut contenir une vingtaine de tsé-tsé ; elle est aussi d'unc manipulation plus facile surtout pour l'alimentation des mouches. Une dizaine de cages soit 200 mouches nécessiteront un cubage de 15 décimétres cubes environ, 400 mouches 30 décimètres cubes et 600 mouches, 45 décimètres cubes (Le récipient contenant lc mélange eau-glycérine 
aura une capacité proportionnelle, approximativement de $1 / 1 / 2$ à 4 |1/2). Pour que l'air soit renouvelé 10 fois en une heure cela nécessiterait un compresseur débitant 140 d̀ 450 litres à l'heure (un compresseur moyen pour laboratoire débite environ 380 litres à l'heure), mais son fonctionnement est assez bruyant. On évitera pour la canalisation d'air les tuyaux en caoutchour; les tuyaux employés seront en matière plastique (le cooutchouc aux humidités élevées étant toxique pour les tsé-tsé). Eventuellement on pourrait utiliser des bouteilles d'air liquide avec détendeur, mais la consommation serait très élevée.

On peut donc concevoir comme dispositif une armoire vitrée de capacité variable, avec une porte à fermeture hermétique et 2 orifices latéraux pour l'admission ef la sortie de l'air traité, garnie au fond d'une couche de sable pour les larves, au-dessus, sur des grillages, sont disposées les cages Roubaud. On peut également mettre à l'intérieur, d'une façon temporaire ou permanente, des appareils de mesure d'humidité et de tempéroture, appareils de faible encombrement comme un thermomètre à minima et maxima ef un psychromètre à aspiration (J. Richard par exemple).

Les mouches ne seront sorties qu'au. moment des repas. Beaucoup d'espèces tolèrent momentanément une humidité relative de $75 \mathrm{p} .100$.

Telles sont les principales modifications que l'on pourrait apporter au dispositif de Johnson pour l'élevage des tsé-tsé sous les tropiques, car en Europe, une salle disposant d'un chauffage avec thermostat et un système d'humidification avec hygrostat semble d'une réalisation matérielle plus facile.

Institut d'Elevage
et de Médecine vétérinaire
des Pays tropicaux
Laboratoire d'entomologie.

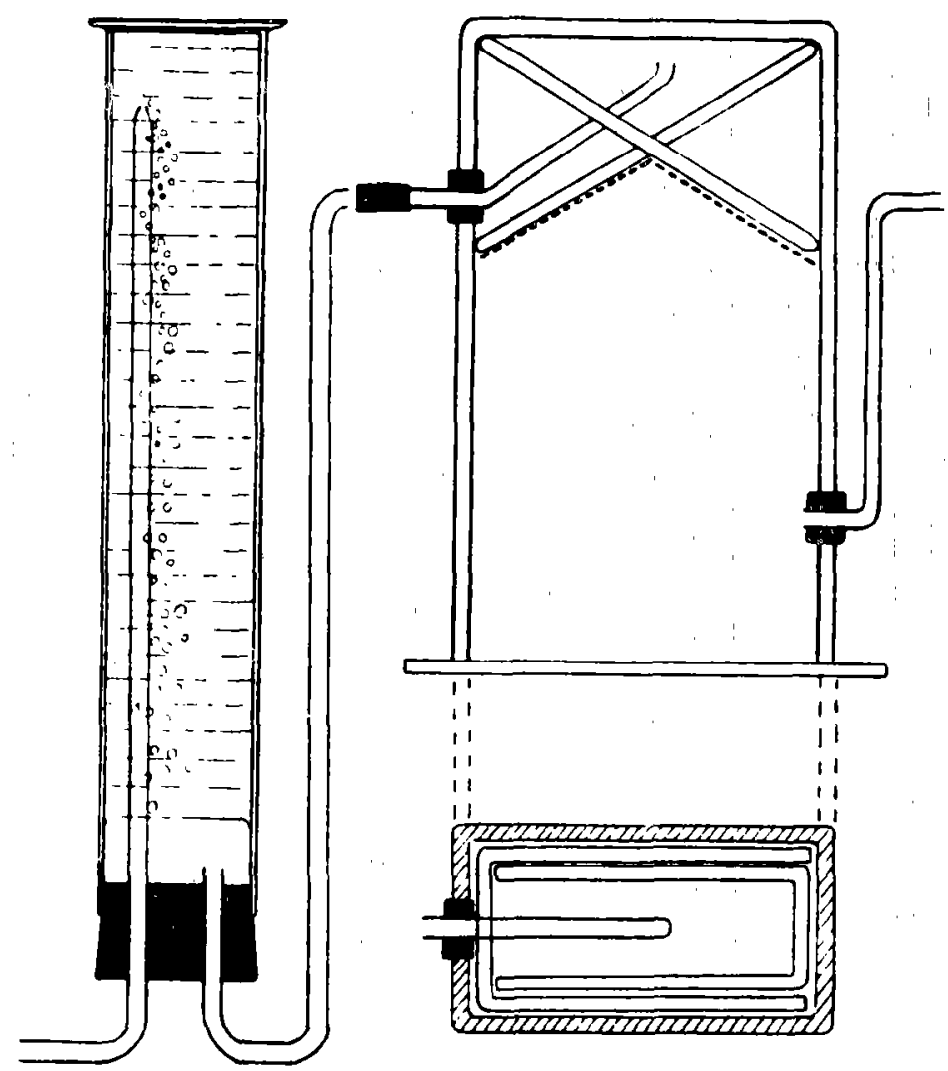

Dispositif d'après Johnson 


\section{SUMMARY}

A Technical Note on Johnson's System of maintaining artificially-bred Insecis at a constant Degree of Humidity. Possibility of applying this Technique to the breeding of Glossinae

The author describes studies on the possibility of breeding glossinae under tropical conditions, using Johnson's technique.

\section{RESUMEN}

Nota técnica sobre el dispositivo de Johnson para el mantenimiento de los insectos de cría a una humedad constante.

Aplicación eventual a una cría de Glossinas.

Fundamentándose en los trabajos de Johnson, el autor examina la posibilidad de practicar la cría de Glossinas en medio tropical por medio del dispositivo descrito.

\section{BIBLIOGRAPHIE}

JOHNSON (C. G.). - The maintenance of the : high atmosphérie humidities for entomological with glycerol-water mixtures (L'entretien d'humidités atmosphériques élevées pour des travaux d'entomologie avec des mélanges glycérine-eau). Am. Appl. Biolog. 1940, $27: 295-99$. 